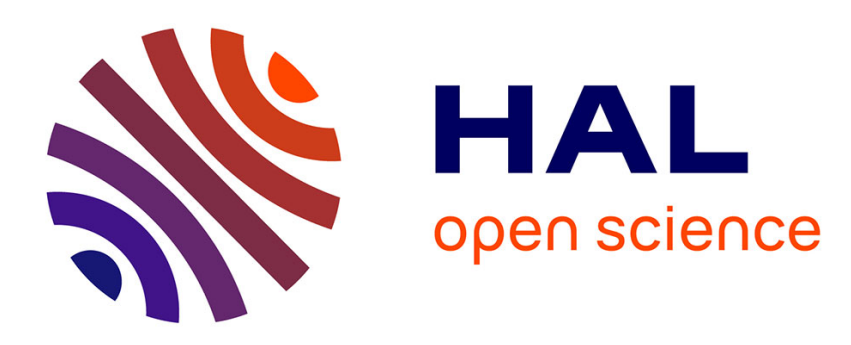

\title{
Incompleteness Theorems, Large Cardinals, and Automata over Infinite Words
}

\author{
Olivier Finkel
}

\section{To cite this version:}

Olivier Finkel. Incompleteness Theorems, Large Cardinals, and Automata over Infinite Words. 42nd International Colloquium on Automata, Languages, and Programming, ICALP 2015, Jul 2015, Kyoto, Japan. pp.222-233, 10.1007/978-3-662-47666-6_18 . hal-01234945

\section{HAL Id: hal-01234945 \\ https://hal.science/hal-01234945}

Submitted on 27 Nov 2015

HAL is a multi-disciplinary open access archive for the deposit and dissemination of scientific research documents, whether they are published or not. The documents may come from teaching and research institutions in France or abroad, or from public or private research centers.
L'archive ouverte pluridisciplinaire HAL, est destinée au dépôt et à la diffusion de documents scientifiques de niveau recherche, publiés ou non, émanant des établissements d'enseignement et de recherche français ou étrangers, des laboratoires publics ou privés. 


\title{
Incompleteness Theorems, Large Cardinals, and Automata over Infinite Words
}

\author{
Olivier Finkel \\ Institut de Mathématiques de Jussieu - Paris Rive Gauche \\ CNRS et Université Paris 7, France. \\ Olivier.Finkel@math. univ-paris-diderot.fr
}

\begin{abstract}
We prove that there exist some 1-counter Büchi automata $\mathcal{A}_{n}$ for which some elementary properties are independent of theories like $T_{n}=:$ ZFC + "There exist (at least) $n$ inaccessible cardinals", for integers $n \geq 1$. In particular, if $T_{n}$ is consistent, then " $L\left(\mathcal{A}_{n}\right)$ is Borel", " $L\left(\mathcal{A}_{n}\right)$ is arithmetical", " $L\left(\mathcal{A}_{n}\right)$ is $\omega$-regular", " $L\left(\mathcal{A}_{n}\right)$ is deterministic", and " $L\left(\mathcal{A}_{n}\right)$ is unambiguous" are provable from $\mathbf{Z F C}$ + "There exist (at least) $n+1$ inaccessible cardinals" but not from ZFC + "There exist (at least) $n$ inaccessible cardinals". We prove similar results for infinitary rational relations accepted by 2 -tape Büchi automata.
\end{abstract}

Keywords: Automata and formal languages; logic in computer science; infinite words; 1 -counter Büchi automaton; 2-tape Büchi automaton; models of set theory; incompleteness Theorems; large cardinals; inaccessible cardinals; independence from the axiomatic system " $\mathbf{Z F C}+$ there exist $n$ inaccessible cardinals".

\section{Introduction}

The theory of automata reading infinite words, which is closely related to infinite games, is now a rich theory which is used for the specification and verification of nonterminating systems, see [GTW02,PP04].

As noticed in [Fin11], some connections between Automata Theory and Set Theory had arosen in the study of monadic theories of well orders, but this was related to automata reading much longer transfinite words than words of length $\omega$ or even than words of length a countable ordinal.

Then one usually thought that the finite or infinite computations appearing in Computer Science are "well defined" in the axiomatic framework of mathematics, and thus that a property on automata is either true or false and that one has not to take care of the different models of Set Theory (except perhaps for the Continuum Hypothesis $\mathbf{C H}$ which is known to be independent from ZFC). And the connections between Automata Theory and Set Theory seemed very far from the practical aspects of Computer Science.

In [Fin09] we recently proved a surprising result: the topological complexity of an $\omega$-language accepted by a 1-counter Büchi automaton, or of an infinitary rational relation accepted by a 2-tape Büchi automaton, is not determined by the axiomatic system ZFC. In particular, there is a 1-counter Büchi automaton $\mathcal{A}$ (respectively, a 2-tape Büchi 
automaton $\mathcal{B}$ ) and two models $\mathbf{V}_{1}$ and $\mathbf{V}_{2}$ of $\mathbf{Z F C}$ such that the $\omega$-language $L(\mathcal{A})$ (respectively, the infinitary rational relation $L(\mathcal{B})$ ) is Borel in $\mathbf{V}_{1}$ but not in $\mathbf{V}_{2}$. We have proved in [Fin 11] other independence results, showing that some basic cardinality questions on automata reading infinite words actually depend on the models of ZFC (see also [Fin10] for similar results for Büchi-recognizable languages of infinite pictures).

The next step in this research project was to determine which properties of automata actually depend on the models of $\mathbf{Z F C}$, and to achieve a more complete investigation of these properties.

We obtain in this paper some more independence results which are more general and are related to the consistency of theories which are recursive extensions of the theory $\mathbf{Z F C}$ (while in the two papers [Fin09,Fin11] the independence results depended on the value of the ordinal $\omega_{1}^{\mathbf{L}}$ which plays the role of the first uncountable ordinal in the constructible universe $\mathbf{L}$ ).

Recall that a large cardinal in a model of set theory is a cardinal which is in some sense much larger than the smaller ones. This may be seen as a generalization of the fact that $\omega$ is much larger than all finite cardinals. The inaccessible cardinals are the simplest such large cardinals. Notice that it cannot be proved in ZFC that there exists an inaccessible cardinal, but one usually believes that the existence of such cardinals is consistent with the axiomatic theory ZFC. The assumed existence of large cardinals have many consequences in Set Theory as well as in many other branches of Mathematics like Algebra, Topology or Analysis, see [Jec02].

We prove that there exist some 1-counter Büchi automata $\mathcal{A}_{n}$ for which some elementary properties are independent of theories like $T_{n}=: \mathbf{Z F C}+$ "There exist (at least) $n$ inaccessible cardinals", for integers $n \geq 1$. We first prove that " $L\left(\mathcal{A}_{n}\right)$ is Borel", " $L\left(\mathcal{A}_{n}\right)$ is arithmetical", " $L\left(\mathcal{A}_{n}\right)$ is $\omega$-regular", " $L\left(\mathcal{A}_{n}\right)$ is deterministic", and " $L\left(\mathcal{A}_{n}\right)$ is unambiguous" are equivalent to the consistency of the theory $T_{n}$. This implies that, if $T_{n}$ is consistent, all these statements are provable from $\mathbf{Z F C}+$ "There exist (at least) $n+1$ inaccessible cardinals" but not from $\mathbf{Z F C}+$ "There exist (at least) $n$ inaccessible cardinals". We prove similar results for infinitary rational relations accepted by 2 -tape Büchi automata. Notice that the same reults can be proved for other large cardinals like hyperinaccessible or Mahlo cardinals, see [Jec02] for a precise definition of these cardinals.

The paper is organized as follows. We recall the notion of counter automata in Section 2. We expose some results of Set Theory in Section 3, and we prove our main results about 1-counter $\omega$-languages in Section 4. We prove similar results for infinitary rational relations in Section 5. Concluding remarks are given in Section 6.

\section{Counter Automata}

We assume the reader to be familiar with the theory of formal $(\omega$-)languages [Tho90,Sta97]. We recall the usual notations of formal language theory.

If $\Sigma$ is a finite alphabet, a non-empty finite word over $\Sigma$ is any sequence $x=$ $a_{1} \ldots a_{k}$, where $a_{i} \in \Sigma$ for $i=1, \ldots, k$, and $k$ is an integer $\geq 1$. The length of $x$ is $k$, denoted by $|x|$. The empty word has no letter and is denoted by $\lambda$; its length is $0 . \Sigma^{\star}$ is the set of finite words (including the empty word) over $\Sigma$. 
The first infinite ordinal is $\omega$. An $\omega$-word over $\Sigma$ is an $\omega$-sequence $a_{1} \ldots a_{n} \ldots$, where for all integers $i \geq 1, a_{i} \in \Sigma$. When $\sigma=a_{1} \ldots a_{n} \ldots$ is an $\omega$-word over $\Sigma$, we write $\sigma(n)=a_{n}, \sigma[n]=\sigma(1) \sigma(2) \ldots \sigma(n)$ for all $n \geq 1$ and $\sigma[0]=\lambda$.

The usual concatenation product of two finite words $u$ and $v$ is denoted $u . v$ (and sometimes just $u v$ ). This product is extended to the product of a finite word $u$ and an $\omega$-word $v$ : the infinite word $u . v$ is then the $\omega$-word such that:

$(u . v)(k)=u(k)$ if $k \leq|u|$, and $(u . v)(k)=v(k-|u|)$ if $k>|u|$.

The set of $\omega$-words over the alphabet $\Sigma$ is denoted by $\Sigma^{\omega}$. An $\omega$-language $V$ over an alphabet $\Sigma$ is a subset of $\Sigma^{\omega}$, and its complement (in $\Sigma^{\omega}$ ) is $\Sigma^{\omega}-V$, denoted $V^{-}$.

Let $k$ be an integer $\geq 1$. A $k$-counter machine has $k$ counters, each of which containing a non-negative integer. The machine can test whether the content of a given counter is zero or not. And transitions depend on the letter read by the machine, the current state of the finite control, and the tests about the values of the counters. Notice that in this model some $\lambda$-transitions are allowed.

Formally a $k$-counter machine is a 4-tuple $\mathcal{M}=\left(K, \Sigma, \Delta, q_{0}\right)$, where $K$ is a finite set of states, $\Sigma$ is a finite input alphabet, $q_{0} \in K$ is the initial state, and $\Delta \subseteq K \times(\Sigma \cup$ $\{\lambda\}) \times\{0,1\}^{k} \times K \times\{0,1,-1\}^{k}$ is the transition relation. The $k$-counter machine $\mathcal{M}$ is said to be real time iff: $\Delta \subseteq K \times \Sigma \times\{0,1\}^{k} \times K \times\{0,1,-1\}^{k}$, i.e. iff there are no $\lambda$-transitions.

If the machine $\mathcal{M}$ is in state $q$ and $c_{i} \in \mathbf{N}$ is the content of the $i^{\text {th }}$ counter $\mathcal{C}_{i}$ then the configuration (or global state) of $\mathcal{M}$ is the $(k+1)$-tuple $\left(q, c_{1}, \ldots, c_{k}\right)$.

For $a \in \Sigma \cup\{\lambda\}, q, q^{\prime} \in K$ and $\left(c_{1}, \ldots, c_{k}\right) \in \mathbf{N}^{k}$ such that $c_{j}=0$ for $j \in E \subseteq$ $\{1, \ldots, k\}$ and $c_{j}>0$ for $j \notin E$, if $\left(q, a, i_{1}, \ldots, i_{k}, q^{\prime}, j_{1}, \ldots, j_{k}\right) \in \Delta$ where $i_{j}=0$ for $j \in E$ and $i_{j}=1$ for $j \notin E$, then we write:

$$
a:\left(q, c_{1}, \ldots, c_{k}\right) \mapsto \mathcal{M}\left(q^{\prime}, c_{1}+j_{1}, \ldots, c_{k}+j_{k}\right) .
$$

Thus the transition relation must obviously satisfy:

if $\left(q, a, i_{1}, \ldots, i_{k}, q^{\prime}, j_{1}, \ldots, j_{k}\right) \in \Delta$ and $i_{m}=0$ for some $m \in\{1, \ldots, k\}$ then $j_{m}=0$ or $j_{m}=1$ (but $j_{m}$ may not be equal to -1 ).

Let $\sigma=a_{1} a_{2} \ldots a_{n} \ldots$ be an $\omega$-word over $\Sigma$. An $\omega$-sequence of configurations $r=\left(q_{i}, c_{1}^{i}, \ldots c_{k}^{i}\right)_{i>1}$ is called a run of $\mathcal{M}$ on $\sigma$, iff:

(1) $\left(q_{1}, c_{1}^{1}, \ldots c_{k}^{1}\right)=\left(q_{0}, 0, \ldots, 0\right)$

(2) for each $i \geq 1$, there exists $b_{i} \in \Sigma \cup\{\lambda\}$ such that $b_{i}:\left(q_{i}, c_{1}^{i}, \ldots c_{k}^{i}\right) \mapsto_{\mathcal{M}}$ $\left(q_{i+1}, c_{1}^{i+1}, \ldots c_{k}^{i+\overline{1}}\right)$ and such that $a_{1} a_{2} \ldots a_{n} \ldots=b_{1} b_{2} \ldots b_{n} \ldots$

For every such run $r, \operatorname{In}(r)$ is the set of all states entered infinitely often during $r$.

Definition 1. A Büchi $k$-counter automaton is a 5-tuple $\mathcal{M}=\left(K, \Sigma, \Delta, q_{0}, F\right)$, where $\mathcal{M}^{\prime}=\left(K, \Sigma, \Delta, q_{0}\right)$ is a $k$-counter machine and $F \subseteq K$ is the set of accepting states. The $\omega$-language accepted by $\mathcal{M}$ is:

$L(\mathcal{M})=\left\{\sigma \in \Sigma^{\omega} \mid\right.$ there exists a run $r$ of $\mathcal{M}$ on $\sigma$ such that $\left.\operatorname{In}(r) \cap F \neq \emptyset\right\}$

The class of $\omega$-languages accepted by Büchi $k$-counter automata is denoted $\mathbf{B C L}(k)_{\omega}$. The class of $\omega$-languages accepted by real time Büchi $k$-counter automata will be denoted $\mathbf{r}-\mathbf{B C L}(k)_{\omega}$.

We now recall the definition of classes of the arithmetical hierarchy of $\omega$-languages, see [Sta97]. Let $X$ be a finite alphabet. An $\omega$-language $L \subseteq X^{\omega}$ belongs to the class $\Sigma_{n}$ if and only if there exists a recursive relation $R_{L} \subseteq(\mathbb{N})^{n-1} \times X^{\star}$ such that: 


$$
L=\left\{\sigma \in X^{\omega} \mid \exists a_{1} \ldots Q_{n} a_{n} \quad\left(a_{1}, \ldots, a_{n-1}, \sigma\left[a_{n}+1\right]\right) \in R_{L}\right\},
$$

where $Q_{i}$ is one of the quantifiers $\forall$ or $\exists$ (not necessarily in an alternating order). An $\omega$-language $L \subseteq X^{\omega}$ belongs to the class $\Pi_{n}$ if and only if its complement $X^{\omega}-L$ belongs to the class $\Sigma_{n}$. The class $\Sigma_{1}^{1}$ is the class of effective analytic sets which are obtained by projection of arithmetical sets. An $\omega$-language $L \subseteq X^{\omega}$ belongs to the class $\Sigma_{1}^{1}$ if and only if there exists a recursive relation $R_{L} \subseteq \mathbb{N} \times\{0,1\}^{\star} \times X^{\star}$ such that: $L=\left\{\sigma \in X^{\omega} \mid \exists \tau\left(\tau \in\{0,1\}^{\omega} \wedge \forall n \exists m\left((n, \tau[m], \sigma[m]) \in R_{L}\right)\right)\right\}$.

Then an $\omega$-language $L \subseteq X^{\omega}$ is in the class $\Sigma_{1}^{1}$ iff it is the projection of an $\omega$ language over the alphabet $X \times\{0,1\}$ which is in the class $\Pi_{2}$. The class $\Pi_{1}^{1}$ of effective co-analytic sets is simply the class of complements of effective analytic sets.

Recall that a Büchi Turing machine is just a Turing machine working on infinite inputs with a Büchi-like acceptance condition, and that the class of $\omega$-languages accepted by Büchi Turing machines is the class $\Sigma_{1}^{1}$ of effective analytic sets [Sta97]. On the oher hand, one can construct, using a classical construction (see for instance [HMU01]), from a Büchi Turing machine $\mathcal{T}$, a 2-counter Büchi automaton $\mathcal{A}$ accepting the same $\omega$-language. Thus one can state the following proposition.

Proposition 2. An w-language $L \subseteq X^{\omega}$ is in the class $\Sigma_{1}^{1}$ iff it is accepted by a non deterministic Büchi Turing machine, hence iff it is in the class $\mathbf{B C L}(2)_{\omega}$.

\section{Some Results of Set Theory}

We now recall some basic notions of set theory which will be useful in the sequel, and which are exposed in any textbook on set theory, like [Kun80,Jec02].

The usual axiomatic system $\mathbf{Z F C}$ is Zermelo-Fraenkel system $\mathbf{Z F}$ plus the axiom of choice AC. The axioms of ZFC express some natural facts that we consider to hold in the universe of sets. For instance a natural fact is that two sets $x$ and $y$ are equal iff they have the same elements. This is expressed by the Axiom of Extensionality:

$$
\forall x \forall y[x=y \leftrightarrow \forall z(z \in x \leftrightarrow z \in y)] .
$$

Another natural axiom is the Pairing Axiom which states that for all sets $x$ and $y$ there exists a set $z=\{x, y\}$ whose elements are $x$ and $y$ :

$$
\forall x \forall y[\exists z(\forall w(w \in z \leftrightarrow(w=x \vee w=y)))]
$$

Similarly the Powerset Axiom states the existence of the set $\mathcal{P}(x)$ of subsets of a set $x$. Notice that these axioms are first-order sentences in the usual logical language of set theory whose only non logical symbol is the membership binary relation symbol $\in$. We refer the reader to any textbook on set theory for an exposition of the other axioms of ZFC.

A model $(\mathbf{V}, \in)$ of an arbitrary set of axioms $\mathbb{A}$ is a collection $\mathbf{V}$ of sets, equipped with the membership relation $\in$, where " $x \in y$ " means that the set $x$ is an element of the set $y$, which satisfies the axioms of $\mathbb{A}$. We often say " the model $\mathbf{V}$ " instead of " the $\operatorname{model}(\mathbf{V}, \in)$ ".

We say that two sets $A$ and $B$ have same cardinality iff there is a bijection from $A$ onto $B$ and we denote this by $A \approx B$. The relation $\approx$ is an equivalence relation. Using 
the axiom of choice $\mathbf{A C}$, one can prove that any set $A$ can be well-ordered so there is an ordinal $\gamma$ such that $A \approx \gamma$. In set theory the cardinal of the set $A$ is then formally defined as the smallest such ordinal $\gamma$.

The infinite cardinals are usually denoted by $\aleph_{0}, \aleph_{1}, \aleph_{2}, \ldots, \aleph_{\alpha}, \ldots$ The cardinal $\aleph_{\alpha}$ is also denoted by $\omega_{\alpha}$, when it is considered as an ordinal. The first infinite ordinal is $\omega$ and it is the smallest ordinal which is countably infinite so $\aleph_{0}=\omega$ (which could be written $\omega_{0}$ ). The first uncountable ordinal is $\omega_{1}$, and formally $\aleph_{1}=\omega_{1}$.

Let $\mathbf{O N}$ be the class of all ordinals. Recall that an ordinal $\alpha$ is said to be a successor ordinal iff there exists an ordinal $\beta$ such that $\alpha=\beta+1$; otherwise the ordinal $\alpha$ is said to be a limit ordinal and in this case $\alpha=\sup \{\beta \in \mathbf{O N} \mid \beta<\alpha\}$.

We recall now the notions of cofinality of an ordinal and of regular cardinal which may be found for instance in [Jec02]. Let $\alpha$ be a limit ordinal, the cofinality of $\alpha$, denoted $\operatorname{cof}(\alpha)$, is the least ordinal $\beta$ such that there exists a strictly increasing sequence of ordinals $\left(\alpha_{i}\right)_{i<\beta}$, of length $\beta$, such that $\forall i<\beta \quad \alpha_{i}<\alpha \quad$ and $\sup _{i<\beta} \alpha_{i}=$ $\alpha$. This definition is usually extended to 0 and to the successor ordinals: $\operatorname{cof}(0)=$ 0 and $\operatorname{cof}(\alpha+1)=1$ for every ordinal $\alpha$. The cofinality of a limit ordinal is always a limit ordinal satisfying: $\omega \leq \operatorname{cof}(\alpha) \leq \alpha$. Moreover $\operatorname{cof}(\alpha)$ is in fact a cardinal. A cardinal $\kappa$ is said to be regular iff $\operatorname{cof}(\kappa)=\kappa$. Otherwise $\operatorname{cof}(\kappa)<\kappa$ and the cardinal $\kappa$ is said to be singular.

A cardinal $\kappa$ is said to be a (strongly) inaccessible cardinal iff $\kappa>\omega, \kappa$ is regular, and for all cardinals $\lambda<\kappa$ it holds that $2^{\lambda}<\kappa$, where $2^{\lambda}$ is the cardinal of $\mathcal{P}(\lambda)$.

Recall that the class of sets in a model $\mathbf{V}$ of $\mathbf{Z F}$ may be stratified in a transfinite hierarchy, called the Cumulative Hierarchy, which is defined by $\mathbf{V}=\bigcup_{\alpha \in \mathbf{O N}} \mathbf{V}_{\alpha}$, where the sets $\mathbf{V}_{\alpha}$ are constructed by induction as follows:

(1). $\mathbf{V}_{0}=\emptyset$

(2). $\mathbf{V}_{\alpha+1}=\mathcal{P}\left(\mathbf{V}_{\alpha}\right)$ is the set of subsets of $\mathbf{V}_{\alpha}$, and

(3). $\mathbf{V}_{\alpha}=\bigcup_{\beta<\alpha} \mathbf{V}_{\beta}$, for $\alpha$ a limit ordinal.

It is well known that if $\mathbf{V}$ is a model of $\mathbf{Z F C}$ and $\kappa$ is an inaccessible cardinal in $\mathbf{V}$ then $\mathbf{V}_{\kappa}$ is also a model of $\mathbf{Z F C}$. If there exist in $\mathbf{V}$ at least $n$ inaccessible cardinals, where $n \geq 1$ is an integer, and if $\kappa$ is the $n$-th inaccessible cardinal, then $\mathbf{V}_{\kappa}$ is also a model of $\mathbf{Z F C}+$ "There exist exactly $n-1$ inaccessible cardinals". This implies that one cannot prove in $\mathbf{Z F C}$ that there exists an inaccessible cardinal, because if $\kappa$ is the first inaccessible cardinal in $\mathbf{V}$ then $\mathbf{V}_{\kappa}$ is a model of $\mathbf{Z F C}+$ "There exist no inaccessible cardinals".

We assume the reader to be familiar with basic notions of topology which may be found in [Mos80,LT94,Sta97,PP04]. There is a natural metric on the set $\Sigma^{\omega}$ of infinite words over a finite alphabet $\Sigma$ containing at least two letters which is called the prefix metric and is defined as follows. For $u, v \in \Sigma^{\omega}$ and $u \neq v$ let $\delta(u, v)=2^{-l_{\operatorname{pref}(u, v)}}$ where $l_{\operatorname{pref}(u, v)}$ is the first integer $n$ such that the $(n+1)^{s t}$ letter of $u$ is different from the $(n+1)^{s t}$ letter of $v$. This metric induces on $\Sigma^{\omega}$ the usual Cantor topology in which the open subsets of $\Sigma^{\omega}$ are of the form $W . \Sigma^{\omega}$, for $W \subseteq \Sigma^{\star}$.

Define now the Borel Hierarchy of subsets of $\Sigma^{\omega}$ : 
Definition 3. For a non-null countable ordinal $\alpha$, the classes $\boldsymbol{\Sigma}_{\alpha}^{0}$ and $\boldsymbol{\Pi}_{\alpha}^{0}$ of the Borel Hierarchy on the topological space $\Sigma^{\omega}$ are defined as follows:

$\Sigma_{1}^{0}$ is the class of open subsets of $\Sigma^{\omega}, \Pi_{1}^{0}$ is the class of closed subsets of $\Sigma^{\omega}$,

and for any countable ordinal $\alpha \geq 2$ :

$\Sigma_{\alpha}^{0}$ is the class of countable unions of subsets of $\Sigma^{\omega}$ in $\bigcup_{\gamma<\alpha} \Pi_{\gamma}^{0}$.

$\Pi_{\alpha}^{0}$ is the class of countable intersections of subsets of $\Sigma^{\omega}$ in $\bigcup_{\gamma<\alpha} \Sigma_{\gamma}^{0}$.

The class of Borel sets is $\boldsymbol{\Delta}_{1}^{1}:=\bigcup_{\xi<\omega_{1}} \boldsymbol{\Sigma}_{\xi}^{0}=\bigcup_{\xi<\omega_{1}} \boldsymbol{\Pi}_{\xi}^{0}$, where $\omega_{1}$ is the first uncountable ordinal. The class of Borel subsets of $\Sigma^{\omega}$ is strictly included into the class $\Sigma_{1}^{1}$ of analytic sets which are obtained by projection of Borel sets.

We now define completeness with regard to reduction by continuous functions. For a countable ordinal $\alpha \geq 1$, a set $F \subseteq \Sigma^{\omega}$ is said to be a $\boldsymbol{\Sigma}_{\alpha}^{0}$ (respectively, $\boldsymbol{\Pi}_{\alpha}^{0}, \boldsymbol{\Sigma}_{1}^{1}$ )complete set iff for any set $E \subseteq Y^{\omega}$ (with $Y$ a finite alphabet): $E \in \boldsymbol{\Sigma}_{\alpha}^{0}$ (respectively, $E \in \Pi_{\alpha}^{0}, E \in \Sigma_{1}^{1}$ ) iff there exists a continuous function $f: Y^{\omega} \rightarrow \Sigma^{\omega}$ such that $E=f^{-1}(F)$.

\section{Incompleteness results for 1 -counter $\omega$-languages}

We first recall that a (first-order) theory $T$ in the language of set theory is a set of (firstorder) sentences, called the axioms of the theory. If $T$ is a theory and $\varphi$ is a sentence then we write $T \vdash \varphi$ iff there is a formal proof of $\varphi$ from $T$; this means that there is a finite sequence of sentences $\varphi_{j}, 1 \leq j \leq n$, such that $\varphi_{1} \vdash \varphi_{2} \vdash \ldots \varphi_{n}$, where $\varphi_{n}$ is the sentence $\varphi$ and for each $j \in[1, n]$, either $\varphi_{j}$ is in $T$ or $\varphi_{j}$ is a logical axiom or $\varphi_{j}$ follows from $\varphi_{1}, \varphi_{2}, \ldots \varphi_{j-1}$ by usual rules of inference which can be defined purely syntactically. A theory is said to be consistent iff for no (first-order) sentence $\varphi$ does $T \vdash \varphi$ and $T \vdash \neg \varphi$. If $T$ is inconsistent, then for every sentence $\varphi$ it holds that $T \vdash \varphi$. We shall denote Cons $(\mathrm{T})$ the sentence "the theory $T$ is consistent".

Recall that one can code in a recursive manner the sentences in the language of set theory by finite sequences over a finite alphabet, and then simply over the alphabet $\{0,1\}$, by using a classical Gödel numbering of the sentences. We say that the theory $T$ is recursive iff the set of codes of axioms in $T$ is a recursive set of words over $\{0,1\}$. In that case one can also code formal proofs from axioms of a recursive theory $T$ and then $\operatorname{Cons}(\mathrm{T})$ is an arithmetical statement.

The theory $\mathbf{Z F C}$ is recursive and so are the theories $T_{n}=: \mathbf{Z F C}+$ "There exist (at least) $n$ inaccessible cardinals", for any integer $n \geq 1$.

We now recall Gödel's Second Incompleteness Theorem.

Theorem 4 (Gödel 1931). Let $T$ be a consistent recursive extension of $\mathbf{Z F}$. Then $T \nvdash$ Cons $(T)$.

We now state the following lemmas.

Lemma 5. Let $T$ be a recursive theory in the language of set theory. Then there exists a Büchi Turing machine $\mathcal{M}_{T}$, reading words over a finite alphabet $\Sigma$, such that $L\left(\mathcal{M}_{T}\right)=\Sigma^{\omega}$ iff $T$ is consistent and $L\left(\mathcal{M}_{T}\right)=\emptyset$ iff $T$ is inconsistent. And there exists a Büchi Turing machine $\mathcal{M}_{T}^{\prime}$, reading words over the finite alphabet $\Sigma$, such that $L\left(\mathcal{M}_{T}^{\prime}\right)=\Sigma^{\omega}$ iff $T$ is inconsistent and $L\left(\mathcal{M}_{T}^{\prime}\right)=\emptyset$ iff $T$ is consistent. 
Proof. We first describe informally the behaviour of the machine $\mathcal{M}_{T}$. The machine reads the input word but this does not affect the acceptance or non-acceptance of the word. Essentially the machine works as a program which enumerates all the formal proofs from $T$ and enters each time in an accepting state iff the last sentence of the proof is not the sentence " $\exists x(x \neq x)$ ". If the theory $T$ is consistent the machine will enter infinitely often in an accepting state $q_{f}$ and thus the input $\omega$-word will be accepted since the Büchi acceptance condition will be fulfilled. But if the theory is inconsistent then at some point of the computation the machine sees a proof whose last sentence is actually " $\exists x(x \neq x)$ ". In that case the machine enters in a rejecting state and stays forever in that state, and thus the input $\omega$-word will be rejected.

The machine $\mathcal{M}_{T}^{\prime}$ also works as a program which enumerates all the formal proofs from $T$. But this time it enters in an accepting state only when it sees a formal proof whose last sentence is actually " $\exists x(x \neq x)$ ", and then the machine $\mathcal{M}_{T}^{\prime}$ stays in this accepting state forever. Thus the machine accepts all $\omega$-words if the theory $T$ is inconsistent and accepts not any $\omega$-word if the theory $T$ is consistent.

Lemma 6. Let $T$ be a recursive theory in the language of set theory. Then there exists a Büchi Turing machine $\mathcal{M}_{T}$, reading words over a finite alphabet $\Sigma$, such that $L\left(\mathcal{M}_{T}\right)=\Sigma^{\omega}$ iff $T$ is consistent and $L\left(\mathcal{M}_{T}\right)$ is $\boldsymbol{\Sigma}_{1}^{1}$-complete iff $T$ is inconsistent. And there exists a Büchi Turing machine $\mathcal{M}_{T}^{\prime}$, reading words over the finite alphabet $\Sigma$, such that $L\left(\mathcal{M}_{T}^{\prime}\right)=\Sigma^{\omega}$ iff $T$ is inconsistent and $L\left(\mathcal{M}_{T}^{\prime}\right)$ is $\Sigma_{1}^{1}$-complete iff $T$ is consistent.

Proof. This follows from the above Lemma 5 , from the fact that there exists a $\Sigma_{1}^{1}$ complete $\omega$-language accepted by a Büchi Turing machine (and even by a 1-counter Büchi automaton, see [Fin03]), and from the closure under finite union of the class of $\omega$-languages accepted by non-deterministic Büchi Turing machines.

We now state the following result.

Theorem 7. Let $T$ be a recursive theory in the language of set theory. Then there exists a real-time 1-counter Büchi automaton $\mathcal{A}_{T}$ reading words over a finite alphabet $\Gamma$ such that $L\left(\mathcal{A}_{T}\right)=\Gamma^{\omega}$ iff $T$ is consistent and $L\left(\mathcal{A}_{T}\right)$ is $\boldsymbol{\Sigma}_{1}^{1}$-complete iff $T$ is inconsistent. And there exists a real-time 1-counter Büchi automaton $\mathcal{A}_{T}^{\prime}$ reading words over the finite alphabet $\Gamma$, such that $L\left(\mathcal{A}_{T}^{\prime}\right)=\Gamma^{\omega}$ iff $T$ is inconsistent and $L\left(\mathcal{A}_{T}^{\prime}\right)$ is $\boldsymbol{\Sigma}_{1}^{1}$ complete iff $T$ is consistent.

Proof. Let $T$ be a recursive theory in the language of set theory, and $\mathcal{M}_{T}$ be the Büchi Turing machine, reading words over a finite alphabet $\Sigma$, which is given by Lemma 6 . There exists a 2-counter Büchi automaton $\mathcal{C}_{T}$, such that $L\left(\mathcal{M}_{T}\right)=L\left(\mathcal{C}_{T}\right)$, and which can be effectively constructed from the machine $\mathcal{M}_{T}$.

We now use some constructions which were used in a previous paper [Fin06a] to study the topological properties of context-free $\omega$-languages.

Let $E$ be a new letter not in $\Sigma, S$ be an integer $\geq 1$, and $\theta_{S}: \Sigma^{\omega} \rightarrow(\Sigma \cup\{E\})^{\omega}$ be the function defined, for all $x \in \Sigma^{\omega}$, by:

$\theta_{S}(x)=x(1) \cdot E^{S} \cdot x(2) \cdot E^{S^{2}} \cdot x(3) \cdot E^{S^{3}} \cdot x(4) \ldots x(n) \cdot E^{S^{n}} \cdot x(n+1) \cdot E^{S^{n+1}} \ldots$

We proved in [Fin06a] that if $L \subseteq \Sigma^{\omega}$ is an $\omega$-language in the class $\mathbf{B C L}(2)_{\omega}$ and $k=\operatorname{cardinal}(\Sigma)+2, S=(3 k)^{3}$, then one can effectively construct from a Büchi 
2-counter automaton $\mathcal{C}_{T}$ accepting $L$ a real time Büchi 8-counter automaton $\mathcal{D}_{T}$ such that $L\left(\mathcal{D}_{T}\right)=\theta_{S}(L)$.

On the other hand, it is easy to see that $\theta_{S}\left(\Sigma^{\omega}\right)^{-}=(\Sigma \cup\{E\})^{\omega}-\theta_{S}\left(\Sigma^{\omega}\right)$ is accepted by a real time Büchi 1 -counter automaton. The class $\mathbf{r - B C L}(8)_{\omega}$ is closed under finite union in an effective way and thus $\theta_{S}(L) \cup \theta_{S}\left(\Sigma^{\omega}\right)^{-}$is accepted by a real time Büchi 8-counter automaton $\mathcal{E}_{T}$ which can be effectively constructed from $\mathcal{D}_{T}$.

Let now $K=2 \times 3 \times 5 \times 7 \times 11 \times 13 \times 17 \times 19=9699690$ be the product of the eight first prime numbers. Let $\Gamma^{\prime}=\Sigma \cup\{E\}$. An $\omega$-word $x \in\left(\Gamma^{\prime}\right)^{\omega}$ is coded by the $\omega$-word $h_{K}(x)=A \cdot C^{K} \cdot x(1) \cdot B \cdot C^{K^{2}} \cdot A \cdot C^{K^{2}} \cdot x(2) \cdot B \ldots B \cdot C^{K^{n}} \cdot A \cdot C^{K^{n}} \cdot x(n) \cdot B \ldots$

over the alphabet $\Gamma^{\prime \prime}=\Gamma^{\prime} \cup\{A, B, C\}$, where $A, B, C$ are letters not in $\Gamma^{\prime}$. We proved in [Fin06a] that, from a real time Büchi 8-counter automaton $\mathcal{E}_{T}$ accepting $L\left(\mathcal{E}_{T}\right) \subseteq$ $\left(\Gamma^{\prime}\right)^{\omega}$, one can effectively construct a Büchi 1-counter automaton $\mathcal{G}_{T}$ accepting the $\omega$-language $h_{K}\left(L\left(\mathcal{E}_{T}\right)\right) \cup h_{K}\left(\left(\Gamma^{\prime}\right)^{\omega}\right)^{-}$.

Consider now the mapping $\phi_{K}:\left(\Gamma^{\prime} \cup\{A, B, C\}\right)^{\omega} \rightarrow\left(\Gamma^{\prime} \cup\{A, B, C, F\}\right)^{\omega}$ which is defined by: for all $x \in\left(\Gamma^{\prime} \cup\{A, B, C\}\right)^{\omega}$,

$\phi_{K}(x)=F^{K-1} \cdot x(1) . F^{K-1} \cdot x(2) \ldots F^{K-1} \cdot x(n) \cdot F^{K-1} \cdot x(n+1) \cdot F^{K-1} \ldots$

Then the $\omega$-language $\phi_{K}\left(L\left(\mathcal{G}_{T}\right)\right)=\phi_{K}\left(h_{K}\left(L\left(\mathcal{E}_{T}\right)\right) \cup h_{K}\left(\left(\Gamma^{\prime}\right)^{\omega}\right)^{-}\right)$is accepted by a real time Büchi 1-counter automaton $\mathcal{H}_{T}$ which can be effectively constructed from the Büchi 1-counter automaton $\mathcal{G}_{T}$, [Fin06a]. And we set $\Gamma=\Gamma^{\prime} \cup\{A, B, C, F\}$.

On the other hand, the $\omega$-language $\left(\Gamma^{\prime} \cup\{A, B, C, F\}\right)^{\omega}-\phi_{K}\left(\left(\Gamma^{\prime} \cup\{A, B, C\}\right)^{\omega}\right)$ is $\omega$-regular and we can construct a (1-counter) Büchi automaton accepting it. Then one can effectively construct from $\mathcal{H}_{T}$ a real time Büchi 1-counter automaton $\mathcal{A}_{T}$ accepting the $\omega$-language $\phi_{K}\left(h_{K}\left(L\left(\mathcal{E}_{T}\right)\right) \cup h_{K}\left(\left(\Gamma^{\prime}\right)^{\omega}\right)^{-}\right) \cup \phi_{K}\left((\Gamma \cup\{A, B, C\})^{\omega}\right)^{-}$.

It suffices now to see that we have the two following cases:

If $L\left(\mathcal{M}_{T}\right)=L\left(\mathcal{C}_{T}\right)=\Sigma^{\omega}$, then we have successively the following equalities:

$L\left(\mathcal{E}_{T}\right)=(\Sigma \cup\{E\})^{\omega}=\left(\Gamma^{\prime}\right)^{\omega}, L\left(\mathcal{G}_{T}\right)=\left(\Gamma^{\prime} \cup\{A, B, C\}\right)^{\omega}, L\left(\mathcal{A}_{T}\right)=\left(\Gamma^{\prime} \cup\right.$ $\{A, B, C, F\})^{\omega}=\Gamma^{\omega}$,

And if $L\left(\mathcal{M}_{T}\right)=L\left(\mathcal{C}_{T}\right)$ is $\boldsymbol{\Sigma}_{1}^{1}$-complete, then $L\left(\mathcal{A}_{T}\right)$ is also $\boldsymbol{\Sigma}_{1}^{1}$-complete. This follows from the fact that the mapping $\Psi: \Sigma^{\omega} \rightarrow\left(\Gamma^{\prime} \cup\{A, B, C, F\}\right)^{\omega}$ defined by $\Psi(x)=\phi_{K}\left(h_{K}\left(\theta_{S}(x)\right)\right)$ is continuous and satisfies:

$\forall x \in \Sigma^{\omega}\left[x \in L\left(\mathcal{M}_{T}\right) \Longleftrightarrow \Psi(x) \in L\left(\mathcal{A}_{T}\right)\right]$

Finally the construction of the automaton $\mathcal{A}_{T}^{\prime}$ is very similar except we start from the machine $\mathcal{M}_{T}^{\prime}$ instead of the machine $\mathcal{M}_{T}$.

We now briefly recall a few definitions and facts about automata and $\omega$-languages they accept.

An $\omega$-language $L \subseteq \Gamma^{\omega}$ in $\mathbf{B C L}(1)_{\omega}$ is said to be unambiguous iff there exists a 1-counter Büchi automaton $\mathcal{A}$ such that $L=L(\mathcal{A})$ and every $\omega$-word $x \in \Gamma^{\omega}$ has at most one accepting run by $\mathcal{A}$. In the other case the $\omega$-language is said to be inherently ambiguous. An $\omega$-language $L$ accepted by a 1-counter Büchi automaton (respectively, a Büchi Turing machine) is said to have the maximum degree of ambiguity if for every 1counter Büchi automaton (respectively, Büchi Turing machine) $\mathcal{A}$ such that $L=L(\mathcal{A})$ there exist $2^{\aleph_{0}} \omega$-words having $2^{\aleph_{0}}$ accepting runs by $\mathcal{A}$. Notice that this notion may depend on the accepting device which is used. 
An $\omega$-language accepted by a deterministic 1-counter Büchi (respectively, Muller) automaton is a Borel $\Pi_{2}^{0}$-set (respectively, $\Delta_{3}^{0}$-set); the Muller acceptance condition is stronger than the Büchi acceptance condition. The same result is true for any kinds of automata and in particular for Turing machines, see [Tho90,Sta97,PP04].

We now state the following result.

Theorem 8. Let $T$ be a recursive theory in the language of set theory. Then there exist two real-time 1-counter Büchi automata $\mathcal{A}_{T}$ and $\mathcal{A}_{T}^{\prime}$, reading words over a finite alphabet $\Gamma$, such that $\operatorname{Cons}(T)$ is equivalent to each of the following items:

(1) $L\left(\mathcal{A}_{T}\right)=\Gamma^{\omega} ; \quad$ (2) $L\left(\mathcal{A}_{T}\right)$ is $\omega$-regular; (3) $L\left(\mathcal{A}_{T}\right)$ is deterministic; (4) $L\left(\mathcal{A}_{T}\right)$ is Borel; (5) $L\left(\mathcal{A}_{T}\right)$ is in the Borel class $\boldsymbol{\Sigma}_{\alpha}^{0}$ (for a non-null countable ordinal $\alpha$ ); (6) $L\left(\mathcal{A}_{T}\right)$ is in the Borel class $\Pi_{\alpha}^{0}$ (for a non-null countable ordinal $\alpha$ ); (7) $L\left(\mathcal{A}_{T}\right)$ is unambiguous; (8) $L\left(\mathcal{A}_{T}\right)$ is an arithmetical set; (9) $L\left(\mathcal{A}_{T}\right)$ is an hyperarithmetical set, i.e. an effective $\Delta_{1}^{1}$-set; (10) $L\left(\mathcal{A}_{T}\right)$ is in the arithmetical class $\Sigma_{n}$ (for $\left.n \geq 1\right) ; \quad(11) L\left(\mathcal{A}_{T}\right)$ is in the arithmetical class $\Pi_{n}$ (for $n \geq 1$ );

and also to each of the following items:

(1') $\quad L\left(\mathcal{A}_{T}^{\prime}\right) \neq \Gamma^{\omega} ; \quad\left(2^{\prime}\right) \quad L\left(\mathcal{A}_{T}^{\prime}\right)$ is not $\omega$-regular; $\quad\left(3^{\prime}\right) \quad L\left(\mathcal{A}_{T}^{\prime}\right)$ is not deterministic; (4') $L\left(\mathcal{A}_{T}^{\prime}\right)$ is $\boldsymbol{\Sigma}_{1}^{1}$-complete; (5') $L\left(\mathcal{A}_{T}^{\prime}\right)$ is not Borel; (6') $L\left(\mathcal{A}_{T}^{\prime}\right)$ is not in the Borel class $\boldsymbol{\Sigma}_{\alpha}^{0}$ (for a non-null countable ordinal $\alpha$ ); $\quad\left(7^{\prime}\right) \quad L\left(\mathcal{A}_{T}^{\prime}\right)$ is not in the Borel class $\Pi_{\alpha}^{0}$ (for a non-null countable ordinal $\alpha$ ); $\left(8^{\prime}\right) L\left(\mathcal{A}_{T}^{\prime}\right)$ is inherently ambiguous; (9') $L\left(\mathcal{A}_{T}^{\prime}\right)$ has the maximum degree of ambiguity (for acceptance by 1-counter automata or by Turing machines); (10') $L\left(\mathcal{A}_{T}^{\prime}\right)$ is not an arithmetical set; (11') $L\left(\mathcal{A}_{T}^{\prime}\right)$ is not an hyperarithmetical set; $\left(12^{\prime}\right) \quad L\left(\mathcal{A}_{T}^{\prime}\right)$ is not in the arithmetical class $\Sigma_{n}$ (for $\left.n \geq 1\right)$; (13') $L\left(\mathcal{A}_{T}^{\prime}\right)$ is not in the arithmetical class $\Pi_{n}$ (for $n \geq 1$ );

Proof. The real-time 1-counter Büchi automata $\mathcal{A}_{T}$ and $\mathcal{A}_{T}^{\prime}$ are constructed in the proof of the preceding Theorem 7. It is straightforward to check that the $\omega$-language $\Gamma^{\omega}$ is $\omega$-regular, and even accepted by a deterministic Büchi automaton. Moreover it is in every Borel class and in every arithmetical class. It is also clearly unambiguous since it is deterministic. On the other hand a $\Sigma_{1}^{1}$-complete $\omega$-language is not arithmetical, not hyperarithmetical, and not Borel. It cannot be $\omega$-regular since $\omega$-regular languages are Borel $\boldsymbol{\Delta}_{3}^{0}$-sets. Similarly it is not deterministic since it is not a $\boldsymbol{\Delta}_{3}^{0}$-set. Moreover any $\boldsymbol{\Sigma}_{1}^{1}$-complete $\omega$-language accepted by a 1 -counter Büchi automaton (respectively, a Büchi Turing machine) has the maximum degree of ambiguity, see [Fin14].

Recall that we denote $T_{n}$ the theory $\mathbf{Z F C}+$ "There exist (at least) $n$ inaccessible cardinals", for an integer $n \geq 0$. We can apply the preceding theorem to the theories $T_{n}$ which are recursive, and get the real-time 1-counter Büchi automata $\mathcal{A}_{T_{n}}$ and $\mathcal{A}_{T_{n}}^{\prime}$, which will be simply denoted $\mathcal{A}_{n}$ and $\mathcal{A}_{n}^{\prime}$ in the sequel.

Theorem 9. For every integer $n \geq 0$, there exist two real-time 1-counter Büchi automata $\mathcal{A}_{n}$ and $\mathcal{A}_{n}^{\prime}$, reading words over a finite alphabet $\Gamma$, such that $\operatorname{Cons}\left(T_{n}\right)$ is equivalent to each of the items (1)-(11) and (1')-(13') of the preceding theorem where $\mathcal{A}_{T}$ and $\mathcal{A}_{T}^{\prime}$ are replaced by $\mathcal{A}_{n}$ and $\mathcal{A}_{n}^{\prime}$. In particular, if $\mathbf{Z F C}+{ }^{\text {"TTere exist (at least) }}$ 
$n$ inaccessible cardinals" is consistent, then each of the properties of $\mathcal{A}_{n}$ and $\mathcal{A}_{n}^{\prime}$ given by these items (1)-(11) and (1')-(13') is provable from ZFF + "There exist (at least) $n+1$ inaccessible cardinals" but not from $\mathbf{Z F C}+$ "There exist (at least) $n$ inaccessible cardinals".

Proof. The automata $\mathcal{A}_{n}$ and $\mathcal{A}_{n}^{\prime}$ are given by the preceding theorem applied to the theories $T_{n}$. Recall that one can prove from $\mathbf{Z F C}+$ "There exist (at least) $n+1$ inaccessible cardinals" that if $\kappa$ is the $n+1$-th inaccessible cardinal, then the set $\mathbf{V}_{\kappa}$ of the cumulative hierarchy is also a model of $\mathbf{Z F C}+$ "There exist $n$ inaccessible cardinals". This implies that the theory $\mathbf{Z F C}+$ "There exist $n$ inaccessible cardinals" is consistent and thus this implies also the properties of $\mathcal{A}_{n}$ and $\mathcal{A}_{n}^{\prime}$ given by the items (1)-(11) and (1')-(13'). On the other hand if $T_{n}$ is consistent, then these properties are not provable from $T_{n}$. Indeed $T_{n}$ is then a consistent recursive extension of $\mathbf{Z F C}$ and thus by Gödel's Second Incompleteness Theorem we know that $T_{n} \nvdash \operatorname{Cons}\left(T_{n}\right)$.

\section{Incompleteness results for infinitary rational relations}

We now consider acceptance of binary relations over infinite words by 2-tape Büchi automata, firstly considered by Gire and Nivat in [GN84]. A 2-tape automaton is an automaton having two tapes and two reading heads, one for each tape, which can move asynchronously, and a finite control as in the case of a (1-tape) automaton. The automaton reads a pair of (infinite) words $(u, v)$ where $u$ is on the first tape and $v$ is on the second tape, so that a 2-tape Büchi automaton $\mathcal{B}$ accepts an infinitary rational relation $L(\mathcal{B}) \subseteq \Sigma_{1}^{\omega} \times \Sigma_{2}^{\omega}$, where $\Sigma_{1}$ and $\Sigma_{2}$ are two finite alphabets. Notice that $L(\mathcal{B}) \subseteq \Sigma_{1}^{\omega} \times \Sigma_{2}^{\omega}$ may be seen as an $\omega$-language over the product alphabet $\Sigma_{1} \times \Sigma_{2}$.

We now use a coding we have defined in a previous paper [Fin06b] to study the topological complexity of infinitary rational relations. We first recall a coding of an $\omega$ word over the finite alphabet $\Omega=\Sigma \cup\{A, B, C, E, F\}$, where 0 is assumed to be a letter of $\Sigma$, by an $\omega$-word over the alphabet $\Omega^{\prime}=\Omega \cup\{D\}$, where $D$ is an additionnal letter not in $\Omega$. For $x \in \Omega^{\omega}$ the $\omega$-word $h(x)$ is defined by :

$$
h(x)=D \cdot 0 \cdot x(1) \cdot D \cdot 0^{2} \cdot x(2) \cdot D \cdot 0^{3} \cdot x(3) \cdot D \ldots D \cdot 0^{n} \cdot x(n) \cdot D \cdot 0^{n+1} \cdot x(n+1) \cdot D \ldots
$$

It is easy to see that the mapping $h$ from $\Omega^{\omega}$ into $(\Omega \cup\{D\})^{\omega}$ is injective. Let now $\alpha$ be the $\omega$-word over the alphabet $\Omega^{\prime}$ which is simply defined by:

$$
\alpha=D \cdot 0 \cdot D \cdot 0^{2} \cdot D \cdot 0^{3} \cdot D \cdot 0^{4} \cdot D \ldots D \cdot 0^{n} \cdot D \cdot 0^{n+1} \cdot D \ldots
$$

The following result was proved in [Fin06b].

Proposition 10 ([Fin06b]). Let $L \subseteq \Omega^{\omega}$ be in $\mathbf{r - B C L}(1)_{\omega}$ and $\mathcal{L}=h(L) \cup\left(h\left(\Omega^{\omega}\right)\right)^{-}$. Then $\quad R=\mathcal{L} \times\{\alpha\} \cup\left(\Omega^{\prime}\right)^{\omega} \times\left(\left(\Omega^{\prime}\right)^{\omega}-\{\alpha\}\right)$ is an infinitary rational relation. Moreover one can effectively construct from a real time 1-counter Büchi automaton $\mathcal{A}$ accepting $L$ a 2-tape Büchi automaton $\mathcal{B}$ accepting the infinitary relation $R$.

Using this Proposition 10 and Theorem 7 and a very similar reasoning as in the proofs of Theorems 8 and 9, we can now prove the following results. 
Theorem 11. For every integer $n \geq 0$, there exist two 2-tape Büchi automata $\mathcal{B}_{n}$ and $\mathcal{B}_{n}^{\prime}$, reading words over a finite alphabet $\Omega^{\prime} \times \Omega^{\prime}$, such that $\operatorname{Cons}\left(T_{n}\right)$ is equivalent to each of the following items (1)-(11) and (1')-(13')

(1) $L\left(\mathcal{B}_{n}\right)=\left(\Omega^{\prime}\right)^{\omega} \times\left(\Omega^{\prime}\right)^{\omega} ; \quad$ (2) $L\left(\mathcal{B}_{n}\right)$ is $\omega$-regular; (3) $L\left(\mathcal{B}_{n}\right)$ is deterministic; (4) $L\left(\mathcal{B}_{n}\right)$ is Borel; (5) $L\left(\mathcal{B}_{n}\right)$ is in the Borel class $\boldsymbol{\Sigma}_{\alpha}^{0}$ (for a non-null countable ordinal $\alpha$ ); (6) $L\left(\mathcal{B}_{n}\right)$ is in the Borel class $\boldsymbol{\Pi}_{\alpha}^{0}$ (for a non-null countable ordinal $\alpha$ ); (7) $L\left(\mathcal{B}_{n}\right)$ is unambiguous; (8) $L\left(\mathcal{B}_{n}\right)$ is an arithmetical set; (9) $L\left(\mathcal{B}_{n}\right)$ is an hyperarithmetical set, i.e. an effective $\Delta_{1}^{1}$-set; (10) $L\left(\mathcal{B}_{n}\right)$ is in the arithmetical class $\Sigma_{n}$ (for $\left.n \geq 1\right) ; \quad(11) L\left(\mathcal{B}_{n}\right)$ is in the arithmetical class $\Pi_{n}$ (for $n \geq 1$ );

(1') $L\left(\mathcal{B}_{n}^{\prime}\right) \neq\left(\Omega^{\prime}\right)^{\omega} \times\left(\Omega^{\prime}\right)^{\omega} ; \quad\left(2^{\prime}\right) \quad L\left(\mathcal{B}_{n}^{\prime}\right)$ is not $\omega$-regular; (3') $L\left(\mathcal{B}_{n}^{\prime}\right)$ is not deterministic; (4') $L\left(\mathcal{B}_{n}^{\prime}\right)$ is $\boldsymbol{\Sigma}_{1}^{1}$-complete; (5') $L\left(\mathcal{B}_{n}^{\prime}\right)$ is not Borel; (6') $L\left(\mathcal{B}_{n}^{\prime}\right)$ is not in the Borel class $\boldsymbol{\Sigma}_{\alpha}^{0}$ (for a non-null countable ordinal $\alpha$ ); $\left(7^{\prime}\right) L\left(\mathcal{B}_{n}^{\prime}\right)$ is not in the Borel class $\Pi_{\alpha}^{0}$ (for a non-null countable ordinal $\alpha$ ); $\left(8^{\prime}\right) L\left(\mathcal{B}_{n}^{\prime}\right)$ is inherently ambiguous; (9') $L\left(\mathcal{B}_{n}^{\prime}\right)$ has the maximum degree of ambiguity (for acceptance by 2-tape automata or by Turing machines); $\left(10^{\prime}\right) L\left(\mathcal{B}_{n}^{\prime}\right)$ is not an arithmetical set; (11') $L\left(\mathcal{B}_{n}^{\prime}\right)$ is not an hyperarithmetical set; $\left(12^{\prime}\right) L\left(\mathcal{B}_{n}^{\prime}\right)$ is not in the arithmetical class $\Sigma_{n}($ for $n \geq 1) ; \quad\left(13^{\prime}\right) \quad L\left(\mathcal{B}_{n}^{\prime}\right)$ is not in the arithmetical class $\Pi_{n}$ (for $n \geq 1$ );

In particular, if $\mathbf{Z F C}+$ "There exist (at least) $n$ inaccessible cardinals" is consistent, then each of the properties of $\mathcal{B}_{n}$ and $\mathcal{B}_{n}^{\prime}$ given by these items (1)-(11) and (1')-(13') is provable from $\mathbf{Z F C}+$ "There exist (at least) $n+1$ inaccessible cardinals" but not from ZFC + "There exist (at least) $n$ inaccessible cardinals".

\section{Concluding remarks}

Using similar methods as above in this paper, we can construct, for a given theory $T$ in the language of set theory and a given first-order sentence $\Phi$ in the language of set theory, a 1-counter Büchi automaton (or a 2-tape Büchi automaton) $\mathcal{A}_{1}$ (respectively, $\left.\mathcal{A}_{2}, \mathcal{A}_{3}\right)$ such that $L\left(\mathcal{A}_{1}\right)$ (respectively, $L\left(\mathcal{A}_{2}\right), L\left(\mathcal{A}_{3}\right)$ ) is Borel (and deterministic, $\omega$-regular, unambiguous, ...) if and only if the sentence $\Phi$ is provable from $T$, (respectively, $\neg \Phi$ is provable from $T, \Phi$ is independent from $T$ ).

As an example recall that a famous open problem in Complexity Theory is the following question: "Is $\mathbf{P}$ equal to NP?", see [HMU01]. Notice that "P= NP" can be expressed by a first-order sentence $\Psi$ in the language of set theory. Thus one can construct a 2-tape Büchi automaton $\mathcal{A}_{1}$ (respectively, $\mathcal{A}_{2}, \mathcal{A}_{3}$ ) such that $L\left(\mathcal{A}_{1}\right)$ (respectively, $L\left(\mathcal{A}_{2}\right), L\left(\mathcal{A}_{3}\right)$ ) is Borel if and only if the sentence $\Psi$ is provable from $T$, (respectively, $\neg \Psi$ is provable from $T, \Psi$ is independent from $T$ ). Since the "P=NP?" problem is one of the millennium problems for the solution of which one million dollars is offered by the Clay Institute, this is the sum one can win by proving that the infinitary rational relation $L\left(\mathcal{A}_{1}\right)$ (or $L\left(\mathcal{A}_{2}\right)$ or $L\left(\mathcal{A}_{3}\right)$ ) is Borel !

On the other hand, the results of this paper are true for other large cardinals than inaccessible ones. For instance we can replace inaccessible cardinals by hyperinaccessible, Mahlo, hyperMahlo, measurable, ... (see [Jec02]) and still other ones and obtain similar results. 
Finally we mention that in an extended version of this paper we prove similar independence results for timed automata reading timed words.

\section{References}

[Fin03] O. Finkel. Borel hierarchy and omega context free languages. Theoretical Computer Science, 290(3):1385-1405, 2003.

[Fin06a] O. Finkel. Borel ranks and Wadge degrees of omega context free languages. Mathematical Structures in Computer Science, 16(5):813-840, 2006.

[Fin06b] O. Finkel. On the accepting power of two-tape Büchi automata. In Proceedings of the 23rd International Symposium on Theoretical Aspects of Computer Science, STACS 2006, volume 3884 of Lecture Notes in Computer Science, pages 301-312. Springer, 2006.

[Fin09] O. Finkel. The complexity of infinite computations in models of set theory. Logical Methods in Computer Science, 5(4:4):1-19, 2009.

[Fin10] O. Finkel. Decision problems for recognizable languages of infinite pictures. In Studies in Weak Arithmetics, Proceedings of the International Conference 28th Weak Arithmetic Days, June 17-19, 2009, volume 196 of Publications of the Center for the Study of Language and Information, Lecture Notes, pages 127-151. Stanford University, 2010.

[Fin11] O. Finkel. Some problems in automata theory which depend on the models of set theory. RAIRO - Theoretical Informatics and Applications, 45(4):383-397, 2011.

[Fin14] O. Finkel. Ambiguity of $\omega$-languages of Turing machines. Logical Methods in Computer Science, 10(3:12):1-18, 2014.

[GN84] F. Gire and M. Nivat. Relations rationnelles infinitaires. Calcolo, pages 91-125, 1984.

[GTW02] E. Grädel, W. Thomas, and W. Wilke, editors. Automata, Logics, and Infinite Games: A Guide to Current Research [outcome of a Dagstuhl seminar, February 2001], volume 2500 of Lecture Notes in Computer Science. Springer, 2002.

[HMU01] J. E. Hopcroft, R. Motwani, and J. D. Ullman. Introduction to automata theory, languages, and computation. Addison-Wesley Publishing Co., Reading, Mass., 2001. Addison-Wesley Series in Computer Science.

[Jec02] T. Jech. Set theory, third edition. Springer, 2002.

[Kun80] K. Kunen. Set theory, volume 102 of Studies in Logic and the Foundations of Mathematics. North-Holland Publishing Co., Amsterdam-New York, 1980. An introduction to independence proofs.

[LT94] H. Lescow and W. Thomas. Logical specifications of infinite computations. In J. W. de Bakker, W. P. de Roever, and G. Rozenberg, editors, A Decade of Concurrency, volume 803 of Lecture Notes in Computer Science, pages 583-621. Springer, 1994.

[Mos80] Y. N. Moschovakis. Descriptive set theory. North-Holland Publishing Co., Amsterdam, 1980.

[PP04] D. Perrin and J.-E. Pin. Infinite words, automata, semigroups, logic and games, volume 141 of Pure and Applied Mathematics. Elsevier, 2004.

[Sta97] L. Staiger. $\omega$-languages. In Handbook of formal languages, Vol. 3, pages 339-387. Springer, Berlin, 1997.

[Tho90] W. Thomas. Automata on infinite objects. In J. van Leeuwen, editor, Handbook of Theoretical Computer Science, volume B, Formal models and semantics, pages 135191. Elsevier, 1990. 